\title{
Heavy-heavy and heavy-light quarks interactions generated by QCD vacuum
}

\author{
Mirzayusuf Musakhanov ${ }^{1, a}$ \\ ${ }^{1}$ National University of Uzbekistan
}

\begin{abstract}
The QCD vacuum is populated by instantons that correspond to the tunneling processes in the vacuum. This mechanism creates the strong vacuum gluon fields. As result, the QCD vacuum instantons induce very strong interactions between light quarks, initially almost massless. Such a strong interactions bring a large dynamical mass $M$ of the light quarks and bound them to produce almost massless pions in accordance with the spontaneous breaking of the chiral symmetry (SBCS). On the other hand, the QCD vacuum instantons also interact with heavy quarks and responsible for the generation of the heavy-heavy and heavy-light quarks interactions, with a traces of the SBCS. If we take the average instanton size $\bar{\rho}=0.33 \mathrm{fm}$, and the average inter-instanton distance $\bar{R}=1 \mathrm{fm}$ we obtain the dynamical light quark mass to be $M=365 \mathrm{MeV}$ and the instanton media contribution to the heavy quark mass $\Delta M=70 \mathrm{MeV}$. These factors define the coupling between heavy-light and heavy-heavy quarks induced by the QCD vacuum instantons.

We consider first the instanton effects on the heavy-heavy quarks potential, including its spin-dependent part. We also discuss those effects on the masses of the charmonia and their hyperfine mass splittings. At the second part we discuss the interaction between a heavy and light quarks generated by instantons and it's effects.
\end{abstract}

\section{Introduction}

QCD vacuum has a rich topological properties. We know from [1] that the classical potential energy of the gluon field $A$ is periodic along their collective coordinate so-called Chern-Simons number $N_{C S}=\int d^{3} x K_{4}$ (where topological current $K_{\mu}=\frac{1}{16 \pi^{2}} \epsilon_{\mu \alpha \beta \gamma}\left(A_{\alpha}^{a} \partial_{\beta} A_{\gamma}^{a}+\frac{1}{3} \epsilon^{a b c} A_{\alpha}^{a} A_{\beta}^{b} A_{\gamma}^{c}\right)$ ). and oscillator-like in all other directions in functional space. Quasi-classical quantization leads to the band structure. The width of the band is defined by the amplitude of tunneling $\sim e^{-S_{I}}$, where the action $S_{I}$ is calculated on Euclidean classical trajectory between nearest minima - QCD (anti)instanton. It means that (anti)instanton's topological charge $Q_{T}=\frac{1}{32 \pi^{2}} \int d^{4} x F_{\mu \nu}^{a} \tilde{F}_{\mu \nu}^{a}=N_{C S}(+\infty)-N_{C S}(-\infty)= \pm 1$. Here $F_{\alpha \beta} \equiv \frac{1}{i g}\left[i D_{\alpha}, i D_{\beta}\right], \quad i D_{\alpha} \equiv i \partial_{\alpha}+g A_{\alpha}, \quad \tilde{F}_{\mu \nu}^{a} \equiv \frac{1}{2} \epsilon_{\mu v \alpha \beta} F_{\alpha \beta}^{a}$. Then, we may assume that in QCD vacuum 4-dim space is filled by a tunneling processes with the density $n=1 / R^{4}$ and time-space size $\rho$. It correspond to the collection of a (anti)instantons with sizes $\rho$ occupied Euclidean space with interinstanton distances $R$ and total vacuum gluon field is given by $A_{\mu}(x)=\sum_{+}^{N_{+}} A_{\mu}^{I}\left(\zeta_{+}, x\right)+\sum_{-}^{N_{-}} A_{\mu}^{A}\left(\zeta_{-}, x\right)$, where $A_{\mu}^{I}\left(\zeta_{+}, x\right)\left(A_{\mu}^{A}\left(\zeta_{-}, x\right)\right)$ is (anti)instanton - BPST selfdual classical solution of the equations of

\footnotetext{
a e-mail: musakhanov@gmail.com
} 
motion in Euclidean space [2]. Its collective coordinates are $\zeta=(\rho, z, U)-$ size, position and color orientation respectively.

In general, $A_{\mu}(x)$ is a not a solution of the equations of motion and it leads to the interactions between instantons. The quantization on this background also is another source of the inter-instanton interaction. The main assumption of the Instanton Liquid Model (ILM) that these factors stabilize the $\rho$ and $R$. The estimates of the averaged instanton size $\bar{\rho}$; and averaged inter-instanton distance $\bar{R}$ show

$$
\begin{aligned}
& \bar{\rho} \simeq 0.33 \mathrm{fm}, \bar{R} \simeq 1 \mathrm{fm}, \text { (phenomenological) }[3,4], \\
& \bar{\rho} \simeq 0.35 \mathrm{fm}, \bar{R} \simeq 0.95 \mathrm{fm}, \text { (variational) }[3], \\
& \bar{\rho} \simeq 0.36 \mathrm{fm}, \bar{R} \simeq 0.89 \mathrm{fm}, \text { (lattice) [5], } \\
& \bar{\rho} \simeq 0.35 \mathrm{fm}, \bar{R} \simeq 0.86 \mathrm{fm}, \text { (ILM with } 1 / N_{c} \text { corrections) [6]. }
\end{aligned}
$$

They coincide within $10-15 \%$ uncertainty. With packing parameter $\pi^{2}\left(\frac{\bar{\rho}}{\bar{R}}\right)^{4} \sim 0.1-0.3$ we have a comfortable possibility of independent averaging over instanton positions and orientations.

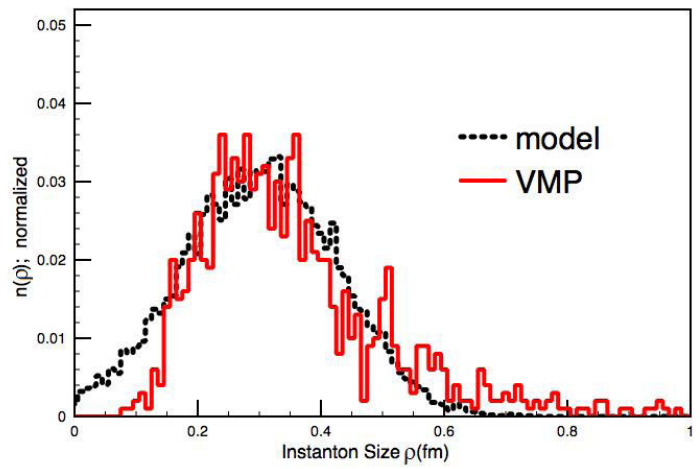

Figure 1. Instanton size distribution from lattice vs ILM [7].

It is clear that in ILM it was neglected by possible portion of large instantons providing confinement (see Fig. 1). The progress with understanding of the confinement is related with extension of a BPST instantons to a KvBLL instantons, which are described by superposition of a dyons [8]. Then, the ILM is extended to the Dyon Liquid Model (DLM) [9, 10]. At SU(2) there is two types of dyons. At large separations $r$ the action density is a clear superposition of these dyons, while at small separations they merge and become like instanton. The KvBLL instanton size and the dyons separation are related by $\pi T \rho^{2}=r$, where $T$ is temperature. The estimate within DLM leads the KvBLL instanton average size $\bar{\rho} \approx \frac{\alpha_{s} N_{c}}{2 \pi \Lambda_{P V}} \approx 0.5 \mathrm{fm}$ at $N_{c}=3, \alpha_{s}=0.5, \Lambda_{P V}=200 \mathrm{MeV}$ [9].

On the other hand, quark core sizes of lowest states of light and heavy quarks hadrons are small. The estimates of the sizes of lowest states of a heavy quarkonium within non-relativistic potential model [11] are demonstrated at Table 1.

Table 1. Quarkonium states and its sizes in non-relativistic potential model [11].

\begin{tabular}{|c|c|c|c|c|c|c|}
\hline State & $J / \psi$ & $\chi_{c}$ & $\psi^{\prime}$ & $\Upsilon$ & $\chi_{b}$ & $\Upsilon^{\prime}$ \\
\hline mass $[\mathrm{Gev}]$ & 3.07 & 3.53 & 3.68 & 9.46 & 9.99 & 10.02 \\
\hline size $r[\mathrm{fm}]$ & 0.25 & 0.36 & 0.45 & 0.14 & 0.22 & 0.28 \\
\hline
\end{tabular}

The same conclusion is on the nucleon. A model estimates from various nucleon form-factors data show nucleon quark core size $r_{N} \sim 0.3-0.5 \mathrm{fm}$ [12].

Since, small quark core size hadrons are insensitive to the confinement, we may safely apply ILM. 


\subsection{Light quarks physics}

We assume that light quark current mass $m$ is small. It means $m \bar{\rho}<<1$. Than zero-mode $\mid \Phi_{0 i}>$, obtained from the Dirac Eq. $(\hat{p}+g \hat{A}) \mid \Phi_{0 i}>=0$, become dominant component in single instanton field light quark propagator $S_{i}$. Accordingly the interpolation formula $[6,13]$ :

$$
S_{i}=S_{0}+S_{0} \hat{p} \frac{\left|\Phi_{0 i}><\Phi_{0 i}\right|}{c_{i}} \hat{p} S_{0}, \quad S_{0}=\frac{1}{\hat{p}+i m}, \quad c_{i}=i m<\Phi_{0 i}\left|\hat{p} S_{0}\right| \Phi_{0 i}>
$$

The advantage of this interpolation is shown by the projection of $S_{i}$ to the zero-modes:

$$
S_{i}\left|\Phi_{0 i}>=\frac{1}{i m}\right| \Phi_{0 i}>, \quad<\Phi_{0 i}\left|S_{i}=<\Phi_{0 i}\right| \frac{1}{i m}
$$

as it must be, while the similar projection of $S_{i}$ given by Ref. [14] has a wrong component, negligible only in the $m \rightarrow 0$ limit. This interpolation well reproduce exact solution [15].

Summing the re-scattering series we arrive to the total ILM light quark propagator:

$$
S=S_{0}-S_{0} \sum_{i, j} \hat{p}\left|\Phi_{0 i}\right\rangle\left\langle\Phi_{0 i}\left|\left(\frac{1}{B(m)}\right)\right| \Phi_{0 j}\right\rangle\left\langle\Phi_{0 j}\right| \hat{p} S_{0}, B(m)=\hat{p} S_{0} \hat{p} .
$$

Our aim is to get light quark determinant represented as $\operatorname{Det}(\hat{p}+g \hat{A}+i m)=\operatorname{Det}_{\text {high }} \cdot \operatorname{Det}_{\text {low }}$, where Det $_{\text {high }}$ receive a contribution from fermion modes with Dirac eigenvalues from the interval $M_{1}$ to the Pauli-Villars mass $M_{P V}$, and Det low $_{\text {low }}$ is accounted eigenvalues less than $M_{1}$. The product of these determinants is independent of the scale $M_{1}$. However, we may calculate both of them only approximately. The quality of the approach is given by the dependence of the product on $M_{1}$, which serves a check of the approximations [14].

Starting from Eq. (4) we were able [13] to find low-frequencies part of light quark determinant in the presence of the light quark sources $(\xi, \xi \dagger)$, which is

$$
\begin{gathered}
\operatorname{Det}_{\text {low }} \exp \left(-\xi^{\dagger} S \xi\right)= \\
=\int \prod_{f} D \psi_{f} D \psi_{f}^{\dagger} \exp \int\left(\psi_{f}^{\dagger}\left(\hat{p}+i m_{f}\right) \psi_{f}+\psi_{f}^{\dagger} \xi_{f}+\xi_{f}^{\dagger} \psi_{f}\right) \prod_{f}\left\{\prod_{ \pm}^{N_{ \pm}} V_{ \pm, f}\left[\psi^{\dagger}, \psi\right]\right\}, \\
V_{ \pm, f}\left[\psi^{\dagger}, \psi\right]=i \int d x\left(\psi_{f}^{\dagger}(x) \hat{p} \Phi_{ \pm, 0}\left(x ; \zeta_{ \pm}\right)\right) \int d y\left(\Phi_{ \pm, 0}^{\dagger}\left(y ; \zeta_{ \pm}\right) \hat{p} \psi_{f}(y)\right) .
\end{gathered}
$$

Fermionic fields $\psi^{\dagger}, \psi$ has a meaning of constituent quarks. Averaging of Eq. (5) over instantons collective coordinates leads to the light quarks partition function:

$$
Z\left[\xi_{f}, \xi_{f}^{\dagger}\right]=\int \prod_{f} D \psi_{f} D \psi_{f}^{\dagger} \exp \int\left(\psi_{f}^{\dagger}\left(\hat{p}+i m_{f}\right) \psi_{f}+\psi_{f}^{\dagger} \xi_{f}+\xi_{f}^{\dagger} \psi_{f}\right) \prod_{ \pm}\left(\overline{\prod_{f} V_{ \pm, f}\left[\psi^{\dagger}, \psi\right]}\right)^{N_{ \pm}}
$$

Small packing parameter provided here independent averaging. The quantity

$$
\overline{\prod_{f} V_{ \pm, f}\left[\psi^{\dagger}, \psi\right]}=\int d \zeta_{ \pm} \prod_{f} V_{ \pm, f}\left[\psi^{\dagger}, \psi\right]
$$

is a non-local t'Hooft-like vertex with $2 N_{f}$-legs, where nonlocality range $\sim \bar{\rho}$. 
at $N_{f}=1$ and $N_{ \pm}=N / 2$ the Eq. (7) leads to

$$
\begin{gathered}
Z\left[\xi, \xi^{\dagger}\right]=e^{-\xi^{\dagger}(\hat{p}+i(m+M(p)))^{-1} \xi} \exp \left[\operatorname{Tr} \ln (\hat{p}+i(m+M(p)))+N \ln \frac{N / 2}{\lambda}-N\right], \\
N=\operatorname{Tr} \frac{i M(p)}{\hat{p}+i(m+M(p))}, \quad M(p)=\frac{\lambda}{N_{c}}(2 \pi \rho F(p))^{2} .
\end{gathered}
$$

Here the form-factor $F(p)$ is given by Fourier-transform of the zero-mode. The coupling $\lambda$ and the dynamical quark mass $M(p)$ are defined by the Eq. (9), where $\operatorname{Tr}(\ldots)=\operatorname{tr}_{c, f, \gamma} \int d^{4} x<x|(\ldots)| x>$.

At $N_{f}>1$ and in the saddle-point approximation (no meson loops contribution) $Z\left[\xi_{f}\right.$, $\left.\xi_{f}^{\dagger}\right]$ has a similar to the Eq. (8) form.

In the lowest order on $1 / N_{c}$ and at $\bar{\rho}=0.33 \mathrm{fm}$ and $\bar{R}=1 \mathrm{fm} M(0) \approx 365 \mathrm{MeV}$, while at $\bar{\rho}=0.36 \mathrm{fm}, \bar{R}=0.89 \mathrm{fm} M(0) \approx 570 \mathrm{MeV}$. Light quark dynamical mass $M$ can be considered as a strength of a light quark-instanton interaction.

Most important features of light hadron physics - Spontaneous Breaking of the Chiral Symmetry (SBCS) and Chiral Perturbation Theory (ChPT) is very well reproduced by the Eqs. (7-9) [6].

\subsection{Heavy quarks in ILM}

The heavy quark Lagrangian is given by $L_{H}=\Psi^{\dagger}\left(\hat{P}+i m_{Q}\right) \Psi$, where $P=p+g A$. We make a FoldyWouthuysen transformation accordingly[16]: $\Psi(x)=\exp \left(-m_{Q} \gamma_{4} x_{4}+O\left(1 / m_{Q}\right)\right) Q(x)$, which leads to

$$
L_{H}=Q^{\dagger} \gamma_{4} P_{4} Q+Q^{\dagger} Q_{1} Q, \quad Q_{1}=\frac{\vec{P}^{2}}{2 m_{Q}}-\frac{\vec{\sigma} \vec{B}}{2 m_{Q}}, \vec{B}=\operatorname{rot} \vec{A} .
$$

Then, (infinitely) heavy quark propagator (Wilson line) in ILM defined as

$$
w=\int D \zeta \frac{1}{\theta^{-1}-\sum_{i} a_{i}},
$$

where

$$
a_{i}(t)=i A_{i, \mu}(x(t)) \frac{d}{d t} x_{\mu}(t), w_{ \pm}=\frac{1}{\theta^{-1}-a_{ \pm}},<t|\theta| t^{\prime}>=\theta\left(t-t^{\prime}\right) .
$$

Pobylitsa [17] derived the Eq. for the quark correlators in instanton vacuum. It's application [18] to the $w$ leads to:

$$
w^{-1}=\theta^{-1}+\sum_{i} \int d \zeta_{i}\left(w-a_{i}^{-1}\right)^{-1} .
$$

In the lowest order on density the Pobylitsa Eq. has a solution

$$
w^{-1}=\theta^{-1}-\frac{N}{2} \sum_{ \pm} \theta^{-1}\left(w_{ \pm}-\theta\right) \theta^{-1}+O\left(N^{2} / V^{2}\right) .
$$

In fact the effective dimensionless parameter of expansion here is $(\bar{\rho} / \bar{R})^{4}$, which is comfortably small.

Instanton media contribution to the heavy quark mass is

$$
\Delta m_{Q}=16 \pi i_{0}(0)\left(\bar{\rho}^{4} / \bar{R}^{4}\right) \bar{\rho}^{-1} / N_{c}, \quad i_{0}(0)=0.55 .
$$

At $\bar{\rho}=0.33 \mathrm{fm}, \bar{R}=1 \mathrm{fm} \Delta m_{Q} \approx 70 \mathrm{MeV}$ and at $\bar{\rho}=0.36 \mathrm{fm}, \bar{R}=0.89 \mathrm{fm} \Delta m_{Q} \approx 140 \mathrm{MeV}$.

$\Delta m_{Q}$ can be considered as a strength of a heavy quark-instanton interaction. 


\section{$2 Q Q$ potential in ILM}

Application of an Eq. similar to the Eq. (12) to the Wilson loop in the lowest order on instanton density leads to the static central potential [18] for the $Q \bar{Q}$ in colorless state

$$
V_{C}(r)=\frac{N}{2 V N_{c}} \sum_{ \pm} \int d^{3} z_{ \pm} \operatorname{tr}_{c}\left[1-P \exp \left(i \int_{L_{1}} d x_{4} A_{ \pm, 4}^{(1)}\right) P \exp \left(-i \int_{L_{2}} d x_{4} A_{ \pm, 4}^{(2)}\right)\right]_{z_{ \pm, 4}=0},
$$

where $L_{1,2}$ are straight lines parallel to the $x_{4}$ and separated by distance $r . A^{(1,2)}$ are the gauge fields projected onto the lines $L_{1,2}$.

Also, from $1 / m_{Q}^{2}$ expansion of the heavy quark propagator [19] it is easy to find spin-dependent parts of the total potential [20]

$$
V(\vec{r})=V_{C}(r)+V_{S S}(r)\left(\vec{S}_{Q} \cdot \vec{S}_{\bar{Q}}\right)+V_{L S}(r)(\vec{L} \cdot \vec{S})+V_{T}(r)\left[3\left(\vec{S}_{Q} \cdot \vec{n}\right)\left(\vec{S}_{\bar{Q}} \cdot \vec{n}\right)-\vec{S}_{Q} \cdot \vec{S}_{\bar{Q}}\right]
$$

where

$$
V_{S S}(r)=\frac{1}{3 m_{Q}^{2}} \nabla^{2} V_{C}(r), \quad V_{L S}(r)=\frac{1}{2 m_{Q}^{2}} \frac{1}{r} \frac{d V_{C}(r)}{d r}, \quad V_{T}(r)=\frac{1}{3 m_{Q}^{2}}\left(\frac{1}{r} \frac{d V_{C}(r)}{d r}-\frac{d^{2} V_{C}(r)}{d r^{2}}\right) .
$$

Fig. 2 represent the result of numerical calculations of the components of colorless state $c \bar{c}$ potential in ILM, corresponding to two sets of the parameters - average instanton size $\bar{\rho}$ and average inter-instanton distance $\bar{R}$.
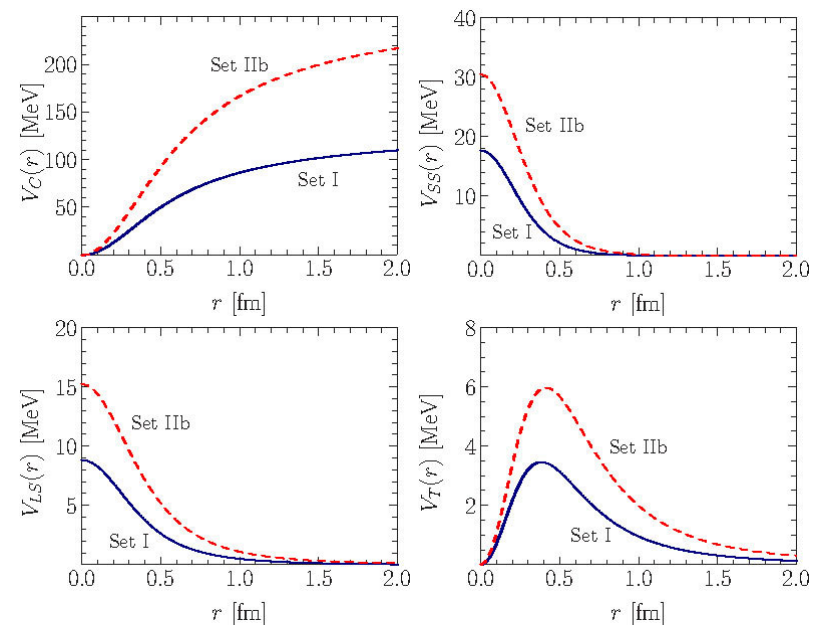

Figure 2. The components of colorless state $c \bar{c}$ potential in ILM. Solid curve $\sim$ Set I $\bar{\rho}=0.33 \mathrm{fm}$ and $\bar{R}=1 \mathrm{fm}$ $\sim$ phenomenology [3, 4]. Dashed one $\sim$ Set IIb $\bar{\rho}=0.36 \mathrm{fm}, \bar{R}=0.89 \mathrm{fm} \sim$ lattice [5] and ILM with $1 / N_{c}$ corrections [6], $m_{c}=1275 \mathrm{MeV}$.

Table 2. ILM contribution to the $(c \bar{c})$ states. $\Delta M_{c \bar{c}}=M_{c \bar{c}}-2 m_{c}$.

\begin{tabular}{c|c|c|c}
$\Delta M_{c \bar{c}}\left(J^{P}\right)$ & Set I & Set IIb & Exp. \\
\hline$\Delta M_{\eta_{c}}\left(0^{-}\right)$ & 118,81 & 203,64 & $433,6 \pm 0.6$ \\
$\Delta M_{J / \psi}\left(1^{-}\right)$ & 119,57 & 205,36 & $546,916 \pm 0.11$ \\
$\Delta M_{\chi_{c 0}}\left(0^{\dagger}\right)$ & 142,43 & 250,86 & $864,75 \pm 0.31$
\end{tabular}


One can see from Table 2 that the instanton effects at lowest states of (cc) quarkonium are not small $\sim 30-40 \%$ in comparison with the experimental data and strongly depend on instanton liquid parameters.

Since DLM pretend to describe QCD vacuum on the large distances too, it is natural to extend these calculations to the whole range of distances at the next step.

\section{Heavy and light quarks in ILM}

In the presence of light quarks the ILM heavy quark propagator is:

$$
\begin{aligned}
& S_{H}=\int \prod_{f} D \psi_{f} D \psi_{f}^{\dagger} \exp \int\left(\psi_{f}^{\dagger}\left(\hat{p}+i m_{f}\right) \psi_{f}\right) \prod_{ \pm}\left(\overline{\prod_{f} V_{ \pm, f}\left[\psi^{\dagger}, \psi\right]}\right)^{N_{ \pm}} w\left[\psi, \psi^{\dagger}\right], \\
& w\left[\psi, \psi^{\dagger}\right]=\left\{\prod_{ \pm}\left(\overline{\prod_{f} V_{ \pm, f}\left[\psi^{\dagger}, \psi\right]}\right)^{N_{ \pm}}\right\}^{-1} \int \prod_{ \pm}^{N_{ \pm}} d \zeta_{ \pm}\left\{\prod_{ \pm}^{N_{ \pm}} \prod_{f} V_{ \pm, f}\left[\psi^{\dagger}, \psi\right]\right\} \frac{1}{\theta^{-1}-\sum_{i} a_{i}} .
\end{aligned}
$$

Obvious extension of the Eq. (12) is

$$
w^{-1}\left[\psi, \psi^{\dagger}\right]=\theta^{-1}+\sum_{i} \int d \zeta_{i}\left(w\left[\psi, \psi^{\dagger}\right]-a_{i}^{-1}\right)^{-1} .
$$

Again, in the lowest order on density we have

$$
\begin{aligned}
& w^{-1}\left[\psi, \psi^{\dagger}\right]=\theta^{-1}+\frac{N}{2} \sum_{ \pm} \frac{1}{\overline{\prod_{f} V_{ \pm, f}\left[\psi^{\dagger}, \psi\right]}} \int d \zeta_{ \pm} \prod_{f} V_{ \pm, f}\left[\psi^{\dagger}, \psi\right]\left(\theta-a_{ \pm}^{-1}\right)^{-1}+O\left(N^{2} / V^{2}\right) \\
& =\theta^{-1}-\frac{N}{2} \sum_{ \pm} \frac{1}{\overline{\prod_{f} V_{ \pm, f}\left[\psi^{\dagger}, \psi\right]}} \Delta_{H, \pm}\left[\psi^{\dagger}, \psi\right]+O\left(N^{2} / V^{2}\right),
\end{aligned}
$$

where

$$
\Delta_{H, \pm}\left[\psi^{\dagger}, \psi\right]=\int d \zeta_{ \pm} \prod_{f} V_{ \pm, f}\left[\psi^{\dagger}, \psi\right] \theta^{-1}\left(w_{ \pm}-\theta\right) \theta^{-1}
$$

represent the interactions of single heavy and $N_{f}$ light quarks induced by (anti)instanton. At $N_{f}=1$ we get the quark propagator in the instanton media with account of light quarks as

$$
S_{H}=\frac{1}{\theta^{-1}-\lambda \sum_{ \pm} \Delta_{H, \pm}\left[\frac{\delta}{\delta \xi}, \frac{\delta}{\delta \xi^{\dagger}}\right]} \exp \left[-\xi^{\dagger}(\hat{p}+i(m+M(p)))^{-1} \xi\right]_{\left.\right|_{\xi=\xi^{\dagger}=0}} .
$$

The heavy and $N_{f}$ light quark interaction term is explicitly given by the expression:

$$
\begin{aligned}
& S_{Q q}=-\lambda \sum_{ \pm} Q^{\dagger} \Delta_{H, \pm}\left[\psi^{\dagger}, \psi\right] Q=-i \lambda \sum_{ \pm} \int d^{4} z_{ \pm} d U_{ \pm} \prod_{f=1}^{N_{f}} \frac{d^{4} k_{f}}{(2 \pi)^{4}} \frac{d^{4} q_{f}}{(2 \pi)^{4}} \exp \left(i\left(q_{f}-k_{f}\right) z_{ \pm}\right) \\
& \times \frac{(2 \pi \rho)^{2} F\left(k_{f}\right) F\left(q_{f}\right)}{8} \psi_{f, a_{f} \alpha_{f}}^{\dagger}\left(k_{f}\right)\left(\gamma_{\mu_{f}} \gamma_{v_{f}} \frac{1 \pm \gamma_{5}}{2}\right)_{\alpha_{f} \beta_{f}}\left(U_{ \pm, i_{f}}^{a_{f}}\left(\tau_{\mu_{f}}^{\mp} \tau_{v_{f}}^{ \pm}\right)_{j_{f}}^{i_{f}} U_{ \pm, b_{f}}^{\dagger j_{f}} \psi_{f, \beta_{f}}^{b_{f}}\left(q_{f}\right)\right. \\
& \times Q_{a_{3}}^{\dagger} U_{ \pm, i_{3}}^{a_{3}}\left(\theta^{-1}\left(w_{ \pm}-\theta\right) \theta^{-1}\right)_{j_{3}}^{i_{3}} U_{ \pm, b_{3}}^{\dagger j_{3}} Q^{b_{3}} .
\end{aligned}
$$

Here matrix $U_{ \pm}$is a (anti)instanton color orientation. It is evident that the integration over $z$ leads to the energy-momentum conservation delta-function, while the integration over color orientation provides the specific structure of the interaction terms. Also, each light quark leg is accompanied by the form-factor $F=F(k \rho)$, which is localized at the region $k \rho \leq 1$, as expected. 


\section{1 $Q Q$ potential generated by light quarks exchange}

The consideration of the $Q \bar{Q}$ correlators (Wilson loops) in the same approach as given by Eqs.(18-19) provide the way for the calculations of $Q \bar{Q}$ potential $V_{l q}$ generated by light quarks exchange. The result of the calculations $V_{l q}(r)$ in simplest case $N_{f}=1$ and with the parameters set $\mathrm{I}$ is shown at Fig. 3.

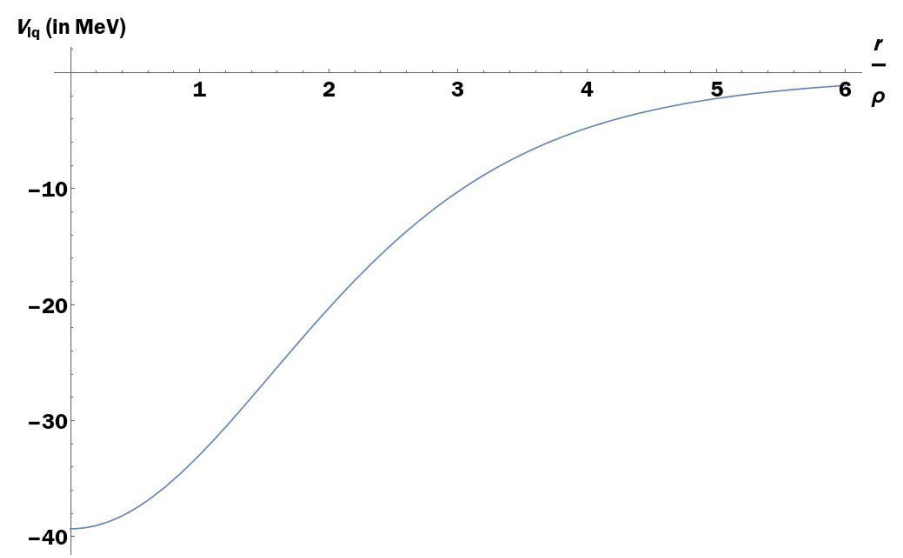

Figure 3. $Q \bar{Q}$ potential $V_{l q}(r / \rho)$ (in MeV) in singlet color state, generated by light quarks exchange $\left(N_{f}=1\right)$. It is taken the parameters set $\mathrm{I} \bar{\rho}=0.33 \mathrm{fm}, \bar{R}=1 \mathrm{fm}$.

It is obvious that at $N_{f}=2$ case we have to have two-pions exchange contribution to the $V_{l q}(r)$.

\subsection{Heavy quark light mesons interaction term}

Heavy quark light quarks interaction term (22) has an essential part which is co-product of colorless heavy and light quarks factors. In the $N_{f}=2$ case it is

$$
\begin{aligned}
& S\left[\psi, \psi^{\dagger}, Q^{\dagger} Q\right]=i \lambda \int d^{4} x \exp (-i p x) \frac{d^{4} p_{1}}{(2 \pi)^{4}} \frac{d^{4} p_{2} d}{(2 \pi)^{4}} \frac{16 \pi \rho^{3}}{N_{c}} i_{0}(p \rho) Q^{\dagger}\left(p_{2}\right) Q\left(p_{1}\right) \\
& \frac{1}{8\left(N_{c}^{2}-1\right)}\left[\left(1-\frac{1}{2 N_{c}}\right)\left(\left(q^{\dagger}(x) q(x)\right)^{2}+\left(q^{\dagger} \gamma_{5} q\right)^{2}-\left(q^{\dagger} \vec{\tau} q\right)^{2}-\left(q^{\dagger} \gamma_{5} \vec{\tau} q\right)^{2}\right)\right. \\
& \left.-\frac{1}{8 N_{c}}\left(\left(q^{\dagger} \sigma_{\mu \nu} q\right)^{2}+\left(q^{\dagger} \gamma_{5} \sigma_{\mu \nu} q\right)^{2}-\left(q^{\dagger} \sigma_{\mu \nu} \vec{\tau} q\right)^{2}-\left(q^{\dagger} \gamma_{5} \sigma_{\mu \nu} \vec{\tau} q\right)^{2}\right)\right]
\end{aligned}
$$

where $i_{0}(y)=\int_{0}^{\infty} d z \frac{\sin [z y]}{z y}\left(z \cos \left[\frac{\pi z}{2 \sqrt{\left(z^{2}+1\right)}}\right]\right)^{2}, q(x)=2 \pi \rho F(i \partial) \psi(x)$ and $q^{\dagger}(x)=2 \pi \rho F(i \partial) \psi^{\dagger}(x)$.

The application of the standard bosonization procedure to the light quarks and the calculation of the path integrals over $\lambda$ and meson fields in the saddle point approximation lead to the vacuum equations in the leading order (LO) on $1 / N_{c}$ expansion. It is natural that these mesons $(\sigma, \vec{\phi}, \ldots)$ have the properties corresponding to the light quarks bilinears $\left(q^{\dagger} q, q^{\dagger} \gamma_{5} \vec{\tau} q, \ldots\right)$.

So, the vacuum equations in the LO are written as

$$
\frac{1}{2} \operatorname{Tr} \frac{i M(p)}{\hat{p}+i(m+M(p))}=N=\frac{1}{2} \sigma_{0}^{2} V, \quad M(p)=M F^{2}(p), \quad M^{2}=(2 \pi \rho)^{4} \lambda \frac{2 N_{c}-1}{2 N_{c}\left(N_{c}^{2}-1\right)} \sigma_{0}^{2} .
$$


They fix the coupling $\lambda$ and the saddle-point $\sigma_{0}$ and accordingly the dynamical quark mass $M(p)$.

Now the total scalar meson field is $\sigma=\sigma_{0}+\sigma^{\prime}$, where $\sigma^{\prime}$ is a quantum fluctuation. Other mesons are presented only by their quantum fluctuations.

At the saddle points we have the effective action for the mesons and colorless heavy quark $Q^{\dagger} Q$ bilinear as

$$
\begin{aligned}
& S\left[\sigma^{\prime}, \vec{\phi}^{\prime}, \eta^{\prime}, \vec{\sigma}^{\prime}, Q^{\dagger} Q\right]=-\operatorname{Tr} \ln \frac{\hat{p}+i(m+M(p))}{\hat{p}+i m}+N / 2+\frac{1}{2} \int d^{4} x\left({\sigma^{\prime 2}}^{2}+{\overrightarrow{\phi^{\prime}}}^{2}+{\overrightarrow{\sigma^{\prime}}}^{2}+{\eta^{\prime}}^{2}\right) \\
& -\operatorname{Tr} \ln \left[1+\frac{1}{\hat{p}+i(m+M(p))} \frac{i M}{\sigma_{0}} F\left(\sigma^{\prime}+i \gamma_{5} \vec{\tau} \vec{\phi}^{\prime}+i \vec{\tau} \vec{\sigma}^{\prime}+\gamma_{5} \eta^{\prime}\right) F\right] \\
& +\operatorname{Tr} \frac{1}{\hat{p}+i(m+M(p))+\frac{i M}{\sigma_{0}} F\left(\sigma^{\prime}+i \gamma_{5} \vec{\tau} \vec{\phi}^{\prime}+i \vec{\tau} \vec{\sigma}^{\prime}+\gamma_{5} \eta^{\prime}\right) F} \\
& \times i\left(M(p)+\frac{M}{\sigma_{0}} F\left(\sigma^{\prime}+i \gamma_{5} \vec{\tau} \vec{\phi}^{\prime}+i \vec{\tau} \vec{\sigma}^{\prime}+\gamma_{5} \eta^{\prime}\right) F\right)\left(\frac{i}{2} \Delta m_{Q} R^{4} \int e^{-i p x} \frac{d^{4} p_{1}}{(2 \pi)^{4}} \frac{d^{4} p_{2}}{(2 \pi)^{4}} \frac{i_{0}(p \rho)}{i_{0}(0)} Q^{\dagger} Q\right) .
\end{aligned}
$$

The first and second lines describe a mesons and their interactions, while the third and forth one describe the renormalization of the heavy quark mass and heavy quark-light quark mesons interactions terms.

From the Eq. (25) we have the heavy quark-pion interaction term [21]:

$$
S_{Q \pi}=i \Delta m_{Q} R^{4} \frac{F_{\pi Q}^{2}}{4} \int d^{4} x \operatorname{tr}_{f} \partial_{\mu} U(x) \partial_{\mu} U^{\dagger}(x) \int e^{-i p x} \frac{d^{4} p_{1}}{(2 \pi)^{4}} \frac{d^{4} p_{2}}{(2 \pi)^{4}} \frac{i_{0}(p \rho)}{i_{0}(0)} Q^{\dagger}\left(p_{2}\right) Q\left(p_{1}\right)
$$

where matrix pion field $U \approx\left(\sigma+i \vec{\tau} \vec{\phi}^{\prime}\right) / \sigma_{0}, F_{\pi Q}^{2} \approx 0.7 F_{\pi}^{2}$ and $p=p_{1}-p_{2}$.

The similar approach for the calculations of $Q \bar{Q}$ correlators (Wilson loop) with account of light quarks will provide the interaction term of the pair of heavy quarks with pions $S_{Q Q \pi}$. Both of these terms $S_{Q Q \pi}$ and $S_{Q \pi}$ are responsible for the two-pions transitions in a heavy quarkoniums. We are planning to apply them in these transitions.

\section{Conclusion}

Lowest state hadrons naturally has a small size quark cores. In this case their properties insensitive to the confinement and ILM is applicable. It was demonstrated by successful application of ILM to the light quark physics. Main feature of light quarks is SBCS naturally described as would-be zero-mode dominance. Light quarks strongly interact with instantons due to zero-modes. Almost massless quark become very massive with dynamical mass $M \sim 365 \mathrm{MeV}$ (at $\bar{\rho}=0.33 \mathrm{fm}, \bar{R}=1 \mathrm{fm}$ ), which can be considered as a strength of a light quark-instanton interaction.

On the other hand, heavy quarks much weaker interact with instantons. The analogous quantity - the shift of a heavy quark mass $\Delta m_{Q} \sim 70 \mathrm{MeV}$ (at the same $\bar{\rho}, \bar{R}$ ) similarly can be considered as a strength of a heavy quark-instanton interaction. Nevertheless the influence of the heavy quarks potential induced by instantons (see Fig. 2) might be important for the spectra of heavy quarkonia as demonstrated by Table 2 .

It is natural that instantons generate also heavy and light quarks interactions with a strength $\sim M \Delta m_{Q}$. Such a vertexes leads to light quark exchange potential for heavy quarks (see Fig. 3). The light-heavy quarks interactions terms naturally leads to the light hadrons transitions in a heavy quarkonia and to the light-heavy quarks bound states - mesons and baryons.

There is a list of a problems which can be solved within this approach. 


\section{References}

[1] L.D. Faddeev, Looking for multi-dimensional solitons in: Non-local Field Theories, Dubna, 1976; R. Jackiw and C. Rebbi, Phys. Rev. Lett. 37(1976)172.

[2] A. Belavin, A. Polyakov, A. Schwartz and Yu. Tyupkin, Phys. Lett. 59(1975)85.

[3] D. Diakonov, Prog. Part. Nucl. Phys. 51(2003)173, arXiv:hep-ph/0212026.

[4] T. Schaefer (INT, Seattle), E. Shuryak (SUNY Stony Brook) Rev.Mod.Phys.70(1998)323, arXiv:hep-ph/9610451.

[5] M. C. Chu, J. M. Grandy, S. Huang and J. W. Negele, Phys. Rev. D 49 (1994) 6039;

J. W. Negele, Nucl. Phys. Proc. Suppl. 73 (1999) 92;

T. DeGrand, Phys. Rev. D 64 (2001) 094508;

P. Faccioli and T. A. DeGrand, Phys. Rev. Lett. 91 (2003) 182001;

P. O. Bowman, U. M. Heller, D. B. Leinweber, A. G. Williams and J. b. Zhang, Nucl. Phys. Proc. Suppl. 128 (2004) 23.

[6] K. Goeke, M. M. Musakhanov and M. Siddikov, Phys. Rev. D 76 (2007) 076007

[7] R. Millo, P. Faccioli, Phys. Rev. D84(2011)034504, arXiv:1105.2163 [hep-ph]

[8] T.C. Kraan and P. van Baal, Phys. Lett. B428(1998)268, arXiv:hep-th/9802049; Nucl. Phys. B533(1998)627, arXiv:hep-th/9805168.

K. Lee and C. Lu, Phys. Rev. D58(1998)025011, arXiv:hep-th/9802108.

[9] D. Diakonov, Nucl. Phys. Proc. Suppl. 195(2009)5, arXiv:0906.2456 [hep-ph].

[10] Yizhuang Liu, E. Shuryak, I. Zahed, Phys. Rev. D92(2015)085006, arXiv:1503.03058 [hep-ph];

Phys. Rev. D92(2015)085007, arXiv:1503.09148 [hep-ph].

[11] S. Digal, O. Kaczmarek, F. Karsch, H. Satz, Eur. Phys. J. C43(2005)71.

[12] Yin He, Fan Wang and Chun Wa Wong, Phys. Lett. B 168(1985)177;

W. Weise, in Quarks and Nuclei (edited by W. Weise), World Scientific, 1985, pp. 57-188;

R.Tegen, in Weak and Electromagnetic Interactions in Nuclei (edited by H. V. Klapdor), Springer Science, 1986, pp.435-440.

[13] M. M. Musakhanov and F. C. Khanna, Phys. Lett. B 395 (1997) 298;

E. D. Salvo and M. M. Musakhanov, Eur. Phys. J. C 5(1998)501;

M. Musakhanov, Eur. Phys. J. C 9 (1999) 235 ;

M. Musakhanov, Nucl. Phys. A 699(2002) 340;

M. M. Musakhanov and H. C. Kim, Phys. Lett. B 572 (2003) 181;

H. C. Kim, M. Musakhanov and M. Siddikov, Phys. Lett. B 608 (2005) 95;

H. C. Kim, M. M. Musakhanov and M. Siddikov, Phys. Lett. B 633 (2006) 701;

K. Goeke, H. C. Kim, M. M. Musakhanov, M. Siddikov, Phys. Rev. D 76 (2007) 116007;

K. Goeke, M. Musakhanov, M. Siddikov, Phys. Rev. D 81 (2010) 054029.

[14] D. Diakonov, V. Polyakov and C. Weiss, Nucl. Phys. B 461 (1996) 539.

[15] R. Carlitz, Chonkyu Lee, Phys. Rev. D17(1978)3238.

[16] S. Chernyshev, M. A. Nowak and I. Zahed, Phys. Rev. D 53 (1996) 5176.

[17] P. V. Pobylitsa, Phys. Lett. B 226 (1989) 387.

[18] D. Diakonov, V. Y. Petrov and P. V. Pobylitsa, Phys. Lett. B 226 (1989) 372.

[19] E. Eichten and F. Feinberg, Phys. Rev. D 23 (1981) 2724.

[20] U. Yakhshiev, Hyun-Chul Kim, B. Turimov, M.M. Musakhanov, Emiko Hiyama, Instanton effects on the heavy-quark static potential, arXiv:1602.06074 [hep-ph].

[21] M. Musakhanov, PoS BaldinISHEPPXXII (2015) 012, arXiv:1412.4472 [hep-ph]. 